Giovanny Miguel Yauri-Santos; Juan Carlos Erazo-Álvarez; Cecilia Ivonne Narváez-Zurita; Verónica Paulina Moreno

http://dx.doi.org/10.35381/r.k.v5i10.700

\title{
Plan de responsabilidad social empresarial para el sector inmobiliario
}

\section{Corporate social responsibility plan for the real estate sector}

\author{
Giovanny Miguel Yauri-Santos \\ giovanny.yauri@psg.ucacue.edu.ec \\ Universidad Católica de Cuenca, Cuenca \\ Ecuador \\ https://orcid.org/0000-0002-9383-1078 \\ Juan Carlos Erazo-Álvarez \\ jcerazo@ucacue.edu.ec \\ Universidad Católica de Cuenca, Cuenca \\ Ecuador \\ https://orcid.org/0000-0001-6480-2270 \\ Cecilia Ivonne Narváez-Zurita \\ inarvaez@ucacue.edu.ec \\ Universidad Católica de Cuenca, Cuenca \\ Ecuador \\ https://orcid.org/0000-0002-7437-9880 \\ Verónica Paulina Moreno \\ veronica.moreno@ucacue.edu.ec \\ Universidad Católica de Cuenca, Cuenca \\ Ecuador \\ https://orcid.org/0000-0003-1517-6124
}

Recibido: 21 de marzo de 2020

Revisado: 04 de abril de 2020

Aprobado: 15 de abril de 2020

Publicado: 19 de mayo de 2020

\section{RESUMEN}

La responsabilidad social empresarial como pacto voluntario de las empresas con la sociedad por ayudar a alcanzar objetivos de sostenibilidad incluyendo ambientales, es un argumento para lograr ventajas empresariales en el mercado. Por lo que el objetivo de la investigación consiste en diseñar un plan de responsabilidad social empresarial para las inmobiliarias de la ciudad de Cuenca. El tipo de investigación fue no experimental, tuvo una orientación cualitativa y cuantitativa y el alcance de estudio fue 
descriptivo-explicativo. Los métodos utilizados fueron el histórico-lógico, analíticosintético, deductivo-inductivo y el método sistémico. Los resultados no sólo demostraron lo elemental que es tener responsabilidad social en las empresas sino también diseñar un plan y socializar a las personas, las mismas que entienden ampliamente el concepto de sostenibilidad y apoyan e idealizan esto en las grandes empresas, sobre todo en las inmobiliarias al ser intermediarias para satisfacer una necesidad primordial.

Descriptores: Responsabilidad social; marketing; ecología; empresa privada. (Palabras tomadas del Tesauro UNESCO).

\section{ABSTRACT}

Corporate social responsibility as a voluntary agreement between companies and society to help achieve sustainability objectives, including environmental ones, is an argument for achieving business advantages in the market. So, the objective of the research is to design a corporate social responsibility plan for real estate companies in the city of Cuenca. The type of research was non-experimental, it had a qualitative and quantitative orientation and the scope of the study was descriptive-explanatory. The methods used were the historical-logical, analytical-synthetic, deductive-inductive and the systemic method. The results not only demonstrated how basic it is to have social responsibility in companies, but also to design a plan and socialize people, the same ones who widely understand the concept of sustainability and support and idealize this in large companies, especially in real estate agents as intermediaries to satisfy a primary need.

Descriptors: Social responsibility; marketing; ecology; private enterprises. (Words taken from the UNESCO Thesaurus).

\section{INTRODUCCIÓN}

En Ecuador el sector inmobiliario se encuentra en una situación de renovación, luego de las crisis económicas de los últimos años; esta depresión se atribuye a la denominada ley de plusvalía, que trajo consigo reducción de recursos destinados para créditos de vivienda, y variación a la baja de los precios en el sector inmobiliario. En este sentido, la demanda de las inmobiliarias fluctúa en cuanto a su tamaño, disponibilidad de créditos hipotecarios, tasas de interés vigentes, precios de las viviendas, monto de las cuotas de entrada, indicador del desempleo, entre otros. Según la presidente de MHeller Group, empresa desarrolladora de proyectos inmobiliarios de distinta índole, entre ellos de vivienda para clase media, manifiesta que: Ecuador está en proceso de recuperación de una crisis importante, por lo que es entendible que el Banco del Instituto Ecuatoriano de Seguridad Social (BIESS) se vea obligado a hacer ajustes de fondo y de forma. 
La actividad de la construcción está estrechamente relacionada con las inmobiliarias, cada una de estas ramas de mercado están presentes a nivel regional y provincial e impulsan la economía, por eso en la provincia del Azuay, se desarrolla con relativa normalidad. Según José Jaramillo, presidente de la Cámara de la Construcción de Cuenca (CCC), afirma que hay una mejoría del 2.5\% al 3\% en el año 2018 en comparación al año 2017; mientras que, Adrián Rodríguez, presidente de la Asociación de Corredores de Bienes Raíces (ACBIR) del Azuay, señala que el incremento es del $5 \%$. La diferencia es obvia, lo que quiere decir que no todo lo que se construye se vende o lo hace con menos celeridad.

La situación de las inmobiliarias, según datos de constructoras (Bermeo asociados), esta con una baja demanda, debido al alto costo de materiales de construcción que son básicos para desarrollar actividades de bienes raíces, entonces las empresas se ven obligadas a la compra y venta en mayor volumen para dinamizar este mercado. Pese a esto al ser elevado el costo de los inmuebles se oferta a un precio elevado para que sus ganancias no se vean afectadas.

En el caso concreto de la ciudad de Cuenca, al igual que Quito, Guayaquil y Ambato, un $80 \%$ de los proyectos inmobiliarios se desarrollan en la parte urbana. En Cuenca sin duda hay casos excepcionales, la ola de extranjeros que buscan un inmueble para habitarlos de forma definitiva hace que las inmobiliarias suban el precio por la capacidad de compra de estos; al ocurrir esta situación se da un desequilibrio ya que la población no puede pagar estas cantidades de dinero. Otro caso significativo son los bienes alrededor de las obras del proyecto tranvía, donde los inmuebles se depreciaron en leve porcentaje. Esto puede tener un efecto contrario al concluir las obras, ya que los precios se podrían disparar por el resultado turístico de este proyecto.

A todo esto, se suma el interés de las inmobiliarias para los proyectos públicos como por ejemplo: casa para todos y vivienda de interés social del gobierno nacional y de la ciudad respectivamente. Cuenca es una de las ciudades más importantes del Ecuador, por lo que se vuelve atractiva para adquirir una propiedad, por su destacado ámbito cultural, social y económico, lo que eleva los precios de una manera desmesurada y claro es una excelente oportunidad para las empresas de esta actividad económica 
incrementen sus ganancia a través de precios elevados, en consecuencia, las personas optan cada vez más por adquirir viviendas en la periferia.

En este orden de ideas, el impacto económico, social y ambiental es clave para una sociedad pues de ello dependerá su futuro, por lo tanto, las empresas tienen la responsabilidad de cumplir este papel, de ahí que todas las organizaciones deben manejar un plan de responsabilidad social empresarial (RSE) en respuesta al impacto de sus decisiones.

Por los antecedentes expuestos, se plantea el siguiente problema de investigación ¿Cómo determinar el impacto de las actividades de bienes raíces en la ciudad de Cuenca en las dimensiones sociales, económicas y medioambientales? ¿Por lo que el objetivo que se establece en la presente investigación consiste en diseñar un plan de responsabilidad social empresarial para el sector inmobiliario que aporte al desarrollo sostenible de la ciudad de Cuenca?

\section{Referencial teórico}

\section{La responsabilidad social empresarial}

La responsabilidad social empresarial (RSE), es un convenio que las empresas de manera espontánea aportan para lograr una sociedad mejor, lo que incluye la conservación del ambiente. La influencia de organismos gubernamentales, como la expedición de directrices de derechos primordiales, ha logrado que las empresas den a conocer su RSE como parte de su filosofía. Los colaboradores y demás grupos de interés (Stakeholders) son beneficiados de la responsabilidad empresarial y a su vez estos tendrán gran influencia en el alcance de objetivos organizacionales. La RSE en sus diferentes conceptos coinciden en la incorporación intencional de las entidades económicas, en enfatizar los problemas de la sociedad y ambientales cuando realicen sus actividades y también su relación con las personas interesadas (Comisión de la Comunidades Europeas, 2001).

Según (Waddock, 2004) la RSE se define como lineamientos de una empresa y sus acciones en función de su impacto en los stakeholders y al ambiente natural. Es la integración de todas las decisiones que tengan repercusiones generales; por lo que no se puede omitir por ser una premisa con bases de una organización social. Sobresale 
este concepto pues la tendencia de las empresas es aplicar estas operaciones como opción para mostrarse como una entidad preocupada por la comunidad, aunque lo sea en menor medida; sin embargo, este es el punto de partida para logra la responsabilidad social que anhelan los interesados e implícitamente los beneficios que las empresas reciben.

La responsabilidad social tiene una concepción ética cuando se habla de organización, que se ve como un todo ante la sociedad, es decir, sus costumbres, actuaciones y resultados, se reflejan en su misión y debe estar ajustadas al entorno. Es así que, los parámetros de la RSE deben tener origen desde el plan estratégico, debe constar en un documento para que no pueda eludirse y tener respaldo de su aplicación, y finalmente debe ser un procedimiento dictado desde los niveles jerárquicos altos y ejecutado por los mismos (Avendaño, 2013). Aquí la comunicación toma un papel vital, pues esta debe reflejar el pensamiento de la empresa como algo rutinario y no casual para evitar el idealismo ante el entorno empresarial. Así, lo ético es comunicar primero su responsabilidad y no ser responsable para luego darlo a conocer (García-Marzá, 2017). (Correa, 2007), refiere a la RSE como una réplica dada por la empresa al ámbito al cual está relacionado, en asuntos de desarrollo personal, laboral de empleados y el efecto positivo en la comunidad. Entonces ser socialmente responsable tiene inmersos aspectos dentro y fuera de la organización; lo interno se connota con el personal, con sus interés propios y también adicionales a la empresa; lo externo está representado por empresas proveedoras, clientes, socios, comunidad en general, a las cuales se debe dar el mismo énfasis que a los internos, pues estos tienen sus perspectivas y deben ser correspondidas de la mejor manera de parte de la empresa.

La teoría que enfatiza el marketing explica que los procedimientos y prácticas de RSE son utilizados para elevar la imagen corporativa y el renombre de las empresas, en la mente de los clientes y/o consumidores, lo que daría como resultado final una ventaja con el resto de organizaciones competidoras. A través de la comunicación de la empresa esto puede masificarse y tener resultados propios de una ventaja competitiva; sin embargo, se desconoce a ciencia cierta la percepción que los grupos de interés tengan al ser informados de estos aspectos, es decir el mayor beneficio lo recibe la 
empresa, pero no siempre serán los mejores en las personas relacionadas de otras formas con la empresa (Alvarado \& Schlesinger, 2008).

(Aguilera \& Puerto, 2012) afirman que la responsabilidad social en empresas tiene una función elemental en lo referente al crecimiento empresarial; esta variable es afectada por diversas causas, la RSE es parte de ellas, pues mejora las decisiones que nacen en la empresa, esto facilita el posicionamiento de los producto o servicio, reinventando la imagen empresarial para tener más clientes y fidelidad de los mismo; entonces mejoran los convenios entre organización y entorno. Así mismo, la responsabilidad con la sociedad aumenta la confianza y notoriedad empresarial de las personas incluidas las de los grupos de interés, por ser interpretado como voluntario, ético y consiente.

Al respecto, (Reyno-Momberg, 2006) asevera que la RSE es un elemento para la administración empresarial, este proceso añade elementos a la filosofía empresarial como factores morales, lineamientos y procedimientos planteados para respaldar las acciones, aumentando los efectos más allá de las empresas y sus intereses económicos, es decir, se extiender al ámbito social y ambiental.

Cabe resaltar que la globalización ha influido en gran medida en las funciones de la empresa, ya que existen nuevas exigencias y desafíos que tienen que afrontar, por los cambios acelerados en el mercado; esto lleva a que acciones que antes eran exigidas ahora sean voluntarias creando un nuevo modelo en la gestión de las organizaciones que genera ventajas competitivas.

De esta manera el análisis en la empresa con respecto a la RSE debe presentarse en todos y cada uno de los componentes de la misma desde su pensamiento organizacional hasta los procesos, de igual forma en las personas desde los niveles operativos hasta los gerenciales. La responsabilidad social en forma global tiene varas concepciones, y su campo de estudio se extiende en varias áreas y se adaptan a las necesidades. Por eso genéricamente la RSE tiene los siguientes tipos:

1. Responsabilidad social empresarial: se refiere al compromiso económico, social y ambiental que las empresas deben tener con toda la sociedad.

2. Responsabilidad social gubernamental: es la competencia en cuanto a respuesta que, de las organizaciones del estado, ante las consecuencias de sus actividades en los distintos colectivos. 
3. Responsabilidad social ambiental: son las medidas que se toman para conservar la naturaleza a futuro, ya sea por parte de una empresa, del gobierno o de forma individual.

4. Responsabilidad social universitaria: es la calidad de profesionales que las universidades dan a la sociedad para que sean pilares fundamentales en el desarrollo local y regional.

5. Responsabilidad social personal o individual: se establece como la responsabilidad de las acciones cada individuo con su entorno, y su influencia ya sea buena o mala en el contexto (Vélez \& Cano, 2016).

La aplicación de la RSE en las empresas depende de varios factores a los cuales hay que considerar y adaptar de acuerdo a la misión de la empresa. (Duarte, 2015) especifica que el tamaño de la empresa no impide la implementación de la RSE, pues todos los procesos pueden generar impactos buenos y malos. Entonces cualquier que sea el caso se debe empezar por lo básico, es decir, la misión de la empresa, sumado a los objetivos en vigencia. Una herramienta del estado de una empresa es el análisis de fortalezas, oportunidades, debilidades y amenazas (FODA), para después plantear estrategias con las dimensiones de la RSE.

Finalmente se crean estrategias para generar valor y por lo tanto sostenibilidad en los periodos actuales y también en los futuros. La inclusión de la teoría a la práctica de la responsabilidad empieza desde el primer eslabón como una actividad base, en la cadena de valor, para desde ahí aportar al objetivo de la responsabilidad sostenible. La actividad empresarial responsable como punto de arranque repercute con la dirección general y se esparcirá hacia las demás áreas de la empresa, por otro lado, (ValarezoGonzález \& Túnez-López, 2014), consideran que la representación de pasos para la implementación de una administración socialmente responsable socialmente se presenta de la siguiente manera:

1. Institucionalización, énfasis y deseo de poner en marcha el proceso de responsabilidad social por parte del nivel jerárquico más alto.

2. Diagnostico interno de la situación actual, con respecto a las actividades realizadas, las repercusiones y percepciones de estas acciones en la sociedad. 
3. Guía de estrategias en la aplicación de planes de gestión empresarial responsable.

4. Socialización interna del plan que se aplicará, incluyendo preparación y motivación.

5. Aplicación y control de la puesta en marcha de lo planificado.

6. Valoración cuantitativa y cualitativa fija.

7. Evaluación externa.

Por su parte, (Cortés-Mura \& Peña-Reyes, 2015), afirman que el objetivo de la RSE busca el balance entre las 3 estructuras, la social, la económica y medioambiental; manteniendo la proyección a futuro dese su origen, de tal manera que a lo largo del tiempo las necesidades sean satisfechas en todo momento. Los ámbitos de la RSE poseen propiedades que se representan en los lineamientos para lograr la sustentabilidad en lo empresarial. Según los objetivos de la empresa y la probabilidad de aplicar las modalidades en las dimensiones de la responsabilidad organizacional se puede tener éxito tanto en el entorno natural como en el económico.

(Cestagalli, Téllez, \& Cuevas, 2016) establecen que la búsqueda de justificar la puesta en marcha de la RSE debe ser una ventaja en el mercado, por la volatilidad de cambios que existe en el ambiente organizacional y que debe tener presente los parámetros de sostenibilidad. Lo económico ya no es el único fin de las empresas, ahora el interés es social para mantener además de una imagen corporativa aceptada un bienestar total con todas las aristas de empresa. Entonces se establece políticas, directrices para satisfacer a todas las partes interesadas y tener un desarrollo que sea sustentado en el bien común.

En palabras de (Molero-García, 2016) lo social considerado como capital en un entorno de RSE es necesario para ejecutar un plan que garantice interacciones con los colaboradores y la comunidad, y claro una disminución de recursos que permita elevar las utilidades. Mientras más estrechas sean las relaciones de la empresa con los actores del mercado cercanos los resultados serán mejores pues se origina un lazo muy superior al del comercio; además la sostenibilidad dependerá mucho de este plan, pues al estar orientado a todas las dimensiones de la RSE la sociedad sabrá ser reciproca con lo entregado en el presente y dará sus frutos en el futuro inmediato o a largo plazo. 
Por otro lado, (Barbachan, 2017) en su investigación menciona que las empresas aun cuando presentan informes públicos sobre su plan de RSE se deben preocupar por el perfeccionamiento de sus procesos, de los objetivos y las maneras de llegar a cumplir, para esto debe incorporar las necesidades de los stakeholders por la importancia de controlar las repercusiones de los planes aplicados. La innovación de todo el proceso es vital para que este no sea estático sino dinámico al momento de ir añadiendo más elementos que permitan fortalecer el plan y cumplirlo de manera más efectiva y eficiente.

Por otro lado, (Duque, Cardona, \& Rendón, 2013), concluyen que la RSE se ha convertido en un elemento clave para el avance de las empresas, esta idea debe consolidarse y sostenerse con bases de reglas, guías y métodos que sirvan para clarificar el concepto de responsabilidad social; además de la necesidad de consensos se establece un patrón que regula de forma minúscula el convenio de empresa con la sociedad. (Caridad, Hernández, \& De-Pelekais, 2014), establecen que existe una empatía de las empresas con la sostenibilidad pues cuando se dan a conocer resultados lo hacen con la premisa de transparencia; con base a esto se pretende tener responsabilidad en el nivel jerárquico más alto como parámetro de sustentabilidad, por último, se estableció que la responsabilidad de gerencia constituye una cadena enlazada con la comunidad y dar el interés social necesario.

Así mismo, (Koehn \& Fierro-Ulloa, 2015) establecen que la gestión de empresas inmobiliarias en relación con la RSE se debate en relación al efecto que este cause, se estudia mediante un análisis a organizaciones inmersas en bienes raíces y que han aplicado con certeza un modelo de responsabilidad empresarial, así mismo se coteja con una empresa nacional que adoptara este modelo; finalmente el buen resultado que se tienen en las partes interesadas con la implementación del plan de RSE, en cuanto a sustentabilidad a través del tiempo y con la legislación en el Ecuador influye en la búsqueda de modelos sociales para sus actividades empresariales. 


\section{La Responsabilidad Social Empresarial en función del impacto económico, social y ambiental}

En este sentido, (Gallardo-Vázquez \& Sánchez-Hernández, 2013), expresan que la RSE es un factor de segundo plano, puesto que se basa en una hipótesis de elementos diferenciados en los 3 ámbitos que conforman la responsabilidad: el ambiental, social y económico. Los parámetros que se analizan tienen que ver en cualquier tipo de empresa, incluyendo las microempresas. Un factor relevante del ámbito social son los colaboradores, en donde se recogen situaciones cotidianas como el ambiente laboral, participación, seguridad familiar y estabilidad. A su vez se refleja en lo externo por ser una fuente de trabajo, atender a grupos vulnerables y sus interacciones con la institucionalidad del Estado.

En este contexto, (Mendoza, Hernández, \& García, 2013) afirman que la responsabilidad en la dimensión social es una cualidad empresarial comprometida con las personas y agrupaciones, las cuales dan un valor a la toma de decisiones de una organización ya sea buena o negativa, con una base de carácter subjetivo y referentes a la legalidad; así se llega a la influencia política pública que genera lineamientos en la relación empresa-comunidad. La responsabilidad social empresarial se connota de varias perspectivas, su cantidad de definiciones, que no sugieren un completo cumplimiento de lo dispuesto por el estado, sino más bien estas tesis se fundamentan en intenciones espontaneas de hacer lo correcto en las actividades empresariales de su entorno.

Según (Avendaño, 2013), las instituciones económicas son fundadoras de una identificación social, por lo tanto, se convierten en organizaciones sociales, puesto que son un cumulo de integración sociocultural que dan como resultado nuevas caracterizaciones, estas mantienen relaciones de tipo activas y notables. La razón social de la empresa pierde tradicionalidad en actividades estratégicas de oportunidad económica, en vez de esto se crean políticas sociales empresariales que soslayan la responsabilidad de las empresas, pues adoptan un papel benéfico para la buena imagen organizacional (González-Bustos, Narváez-Zurita, \& Erazo-Álvarez, 2020).

Por otro lado, (Medina-Giacomozzi \& Severino-González, 2014), afirman que los ámbitos económico, social, legislativo y político forman parte de la estructura actual de 
la empresa; este contexto puede incluir o no a las partes interesadas. Ahora bien, cuando se habla del ámbito económico existen diversos criterios (Chaves-Ávila \& Monzón-Campos, 2018), reflejan en su estudio que la economía en la RSE está en contraposición con el objetivo clásico de las empresas, el mismo que apuntaba a obtener las máximas ganancias monetarias y materiales. Ahora se plantea los objetivos en beneficio a todos, es decir, al bien económico común, de esta manera se establece una política consiente asentada como lineamiento público de regulación de la economía.

El propósito de la responsabilidad económica es instaurar valores en las cartas magnas de las naciones, en el cual se institucionalice para que cada Estado induzca a las empresas a llevar estos valores como bandera económica. Todo esto debe estar sujeto a comprobación desde los contextos pequeños hasta los más grandes y aportar al desarrollo social general. En referencia a esta temática (Andía-Valencia, 2015) sostiene que el área económica esta enlazada con los socios que aportan a la empresa, los cuales esperan por lo menos una utilidad mínima; a más de eso se incorporan los intereses de los demás stakeholders (colaboradores, clientes, proveedores y estado). Es así que los accionistas logran su objetivo aun cuando solo sea lo económico.

Mientras que (Mora-Mayoral \& Martínez-Martínez, 2018), aseguran que la eficiencia y equidad son conceptos de la sustentabilidad económica en las generaciones presentes y futuras, estos dan efectos de riqueza social y con ello se crean directrices que aportan en un plazo mayor a la inmovilidad del buen estilo de vida de la población (GallardoVázquez \& Sánchez-Hernández, 2013), sostienen que la parte económica tiene puntos que enfatizan a los consumidores, que conlleva a calidad en bienes y servicios ofertados; como por ejemplo un servicio post venta. Los proveedores también están inmersos en una administración y estabilidad en cuanto a las relaciones comerciales y a su predilección por la empresa.

Por otro lado, (Henríquez \& Oreste, 2015) afirman que lo primordial en la RSE económica, es tener una organización que certifique una riqueza a largo plazo, que sea estable y que la economía no sea estática. Se reflexiona sobre un equilibrio social con lo monetario manteniendo la sostenibilidad en los recursos. Además de establecen 
parámetros para comparar los efectos en el entorno y en las partes interesadas de la empresa.

Por otra parte, la RSE presenta un amplio estudio de la dimensión ambiental (GallardoVázquez \& Sánchez-Hernández, 2013), afirman que el ámbito ambiental de la responsabilidad empresarial se idealiza desde la reducción al mínimo de los efectos ambientales que el ejercicio económico puede ocasionar, minimizar los recursos energéticos y el resguardo del ecosistema. (Avendaño, 2013) sostiene que la responsabilidad social medioambiental está ligada a parámetros en los que muchos autores coinciden, como por ejemplo la minimización del impacto ecológico, cuidado de los recursos naturales, el progreso en base a la sostenibilidad, el uso de energías amigables con el medio ambiente, difusión de formación y proposición investigativa del tema de conciencia ecológica.

Según (Barbachan, 2017) la teoría sobre la sostenibilidad se entiende como la capacidad de las organizaciones de originar valor monetario conjuntamente con una igualdad social tanto económica como ambiental al realizar sus operaciones; en otras palabras, la sostenibilidad de una empresa se mide de acuerdo con el valor creado para actores internos, externos y ambientales. La eco eficiencia al momento de usar recursos tiene el respaldo de una mayor concienciación ambiental en los procesos de las empresas; la preservación del ámbito natural y su contexto da como resultado la sostenibilidad que se idealiza alcanzar con la responsabilidad empresarial.

Finalmente, (Maldonado-Calero \& Viteri-Martínez, 2017), mencionan que la situación de sostenibilidad es crítica por factores políticos que regulan las actividades económicas, el convenio no consensuado entre empresa-sociedad y el poco aporte académico al tema. Una contracción en el ámbito medioambiental se da por una política gubernamental que no aporta a este aspecto, solo se utilizan lineamientos disfrazados de libertad que no permiten el desarrollo de valores necesarios como la justicia, respeto, solidaridad, etc. Entonces el bienestar será para todo el conjunto de personas, empresas y gobierno, pero no todos contribuyen y esperan los mismos resultados. 


\section{METODOLOGÍA}

La investigación de este trabajo es no experimental puesto que no se manipuló intencionalmente las variables de estudio, sino que se realizó un análisis de estas en su estado natural. Tiene una orientación mixta porque se recolectó datos tanto cualitativos, como cuantitativos. (Hernández, Fernández \& Baptista, 2014) refieren que gran parte los problemas o fenómenos de este tipo requieren una perspectiva constituida por enfoques subjetivos y también por objetivos. El alcance de estudio fue descriptivo explicativo puesto que se detalló las características del objeto de estudio, resaltando las principales y luego se expone las razones por las cuales se dan los hechos que son parte del objeto.

El método histórico - lógico fue parte de la investigación, por tanto, explicó la estructura del trabajo cronológicamente a acuerdo a los sucesos. Otro método utilizado es el analítico-sintético, el analítico fue utilizado porque se realiza un análisis de la información recolectada, así mismo se cumplió con una síntesis de la teoría utilizada para dar información precisa y concreta. Así también, el método deductivo - inductivo fue expuesto en el proceso investigativo. (Del-Cid, Méndez, \& Sandoval, 2011), afirman que se usa este método, debido a que un estudio se realiza de forma circular es decir las dificultades y cifras específicas dan una explicación común; además se obtiene información empírica que revalide dicha explicación y establecer si una hipótesis tiene soporte real.

El método sistémico se aplicó en este trabajo pues utilizó un conjunto de procedimientos y premisas que sirven para dirigir el proceso y aplicar en el desarrollo del tema investigado. Las bases teóricas, la hipótesis, el problema y demás argumentos dieron como resultado un cumulo de conocimiento coherente y concreto; aportó instrumentos para solucionar la problemática planteada en este estudio. Referente a las técnicas utilizadas se estipuló las encuestas para conseguir la información necesaria y así poder examinar las variables planteadas. Con esto, el uso de un cuestionario predefinido y estructurado fue necesario para recoger la información y establecer los resultados del proceso investigativo (Rodríguez, Erazo, \& Narváez, 2019). 


\section{Universo de estudio y tratamiento muestral}

El universo de estudio fue la ciudad de Cuenca, por lo que se consideró la población demográfica, sin embargo, fue necesario estratificar la población para tener resultados más puntuales, ya que no toda la población está inmiscuida en el propósito de este trabajo investigativo. Es así que el universo y tratamiento muestral se desarrolló considerando los datos del Plan de Desarrollo y Ordenamiento Territorial del Cantón Cuenca y se estableció el siguiente procedimiento:

Del total de habitantes de Cuenca, se ha tomado en cuenta factores como zona geográfica, la población económicamente activa y el nivel socioeconómico de la población para definir el universo dando como 88749 (Gobierno Autónomo Descentralizado Municipal del Cantón Cuenca, 2015). Con este resultado se calcula el número de muestras a aplicarse, dando como resultado: 696 encuestas.

\section{Tratamiento estadístico de la información}

La información derivada de la investigación se logró con la aplicación de cuestionarios de los formularios de encuestas de Google (https://docs.google.com/), luego se efectuó el procesamiento de datos obtenidos con la ayuda del programa Microsoft Excel 2016, para mediante este presentar tablas y gráficos que generaron la interpretación efectiva de los resultados obtenidos, mediante la estadística descriptiva.

\section{RESULTADOS}

Los datos resultantes de la investigación de campo, marcan el inicio de la exploración de la situación del sector inmobiliario en relación al tema de la RSE; la información sirve de base para analizar aspectos importantes y tener argumentos al momento de plantear la RSE en una empresa. La estructura de la encuesta ha sido categorizada en tres componentes para su interpretación y se manifiesta de la siguiente manera:

\section{Caracterización de la población}

Lo común de las personas que participaron en la encuesta se presenta en elementos de edad, situación laboral actual y el nivel de ingresos, es así que los resultados arrojan 
que el $36 \%$ de los encuestados tienen entre 31 y 40 años, seguido por un $34 \%$ que tienen entre 18 y 30 años; además el $78 \%$ se encuentran laborando actualmente. También se determina el nivel de ingresos de los encuestados de un rango de $\$ 400$ a $\$ 900$ en un porcentaje de $57 \%$ del total de encuestados y el $22 \%$ que tienen un ingreso entre $\$ 901$ a $\$ 1400$. Esto como consecuencia de que en la actualidad las personas responden a encuestas digitales y es la manera en la que se realizó este procedimiento de recolección de datos.

\section{Conocimiento de RSE en las personas}

En este componente están asignadas cuatro preguntas de la encuesta en donde se establece la definición de RSE por parte de los encuestados que es en un $36 \%$ como ética, seguida de un $29 \%$ que lo define con beneficio social. De igual manera en el conocimiento de las tres dimensiones de la RSE, el 92\% entiende el elemento social, el $97 \%$ la dimensión económica y el $98 \%$ el ambiental, lo que demuestra la plena entereza del tema del grupo de encuestados.

\section{Tabla 1}

Componente conocimiento de RSE en las personas

Pregunta

Respuestas del total de 696 encuestas en porcentaje

Seleccione cuál de los siguientes términos define mejor Ayuda: 8\%

Responsabilidad Social Empresarial para usted.

Cumplimiento: $23 \%$

Ética: 35\%

Beneficio social: $29 \%$

Ninguna: $5 \%$

Cree usted que es deber de las empresas satisfacer las Si: $92 \%$ necesidades de las personas que están relacionadas con la No: $8 \%$ misma.

Cree usted que las empresas deben mejorar la economía Si: $97 \%$ del lugar donde desarrollan sus actividades. No: $3 \%$

Cree usted que es obligación de las empresas colaborar Si: $98 \%$ con el medio ambiente. No: $2 \%$ 
Fuente: Resultados de encuesta

\section{Importancia de la RSE en inmobiliarias}

La importancia de la RSE en las organizaciones está contemplada en las siguientes 6 preguntas, las mismas, se subdividen para diferenciar lo relevante en las personas y lo principal en las empresas. Para las empresas se verifica la interacción de los encuestados con las inmobiliarias, es así que el $45 \%$ lo ha hecho, en tanto que, el $55 \%$ no; a su vez las personas manifiestan en un $94 \%$ que es importante que las inmobiliarias informen que tienen RSE y un $85 \%$ que estas deben tener una sección de la empresa en donde se trate exclusivamente la responsabilidad social.

\section{Tabla 2}

Componente importancia de la RSE en inmobiliarias

Pregunta

\section{Respuestas del total de 696 encuestas en porcentaje}

Ha utilizado o consultado los servicios de una Si: $45 \%$ inmobiliaria en la ciudad de Cuenca. No: $55 \%$

Piensa usted que es importante que las empresas Si: $94 \%$ inmobiliarias informen si tienen Responsabilidad Social No: 6\% Empresarial.

Cree usted que es importante que las inmobiliarias Si: $85 \%$ tengan una sección exclusiva que se encargue de la No: $15 \%$ Responsabilidad Social Empresarial.

Fuente: Resultados de encuesta

Las últimas preguntas se enfocan en lo que la empresa omite de las personas, es decir en sus intereses en cuanto a la RSE. En torno a esto las personas en un $47 \%$ está totalmente de acuerdo en adquirir un servicio inmobiliario si esta demuestra tener RSE, mientras que un $6 \%$ dice que no afecta su decisión aun sabiendo que la empresa sea socialmente responsable. Así mismo el $58 \%$ está totalmente de acuerdo que las empresas manejen un plan de responsabilidad social y finalmente un $67 \%$ está 
totalmente de acuerdo que las inmobiliarias necesariamente deben contar con un plan de RSE

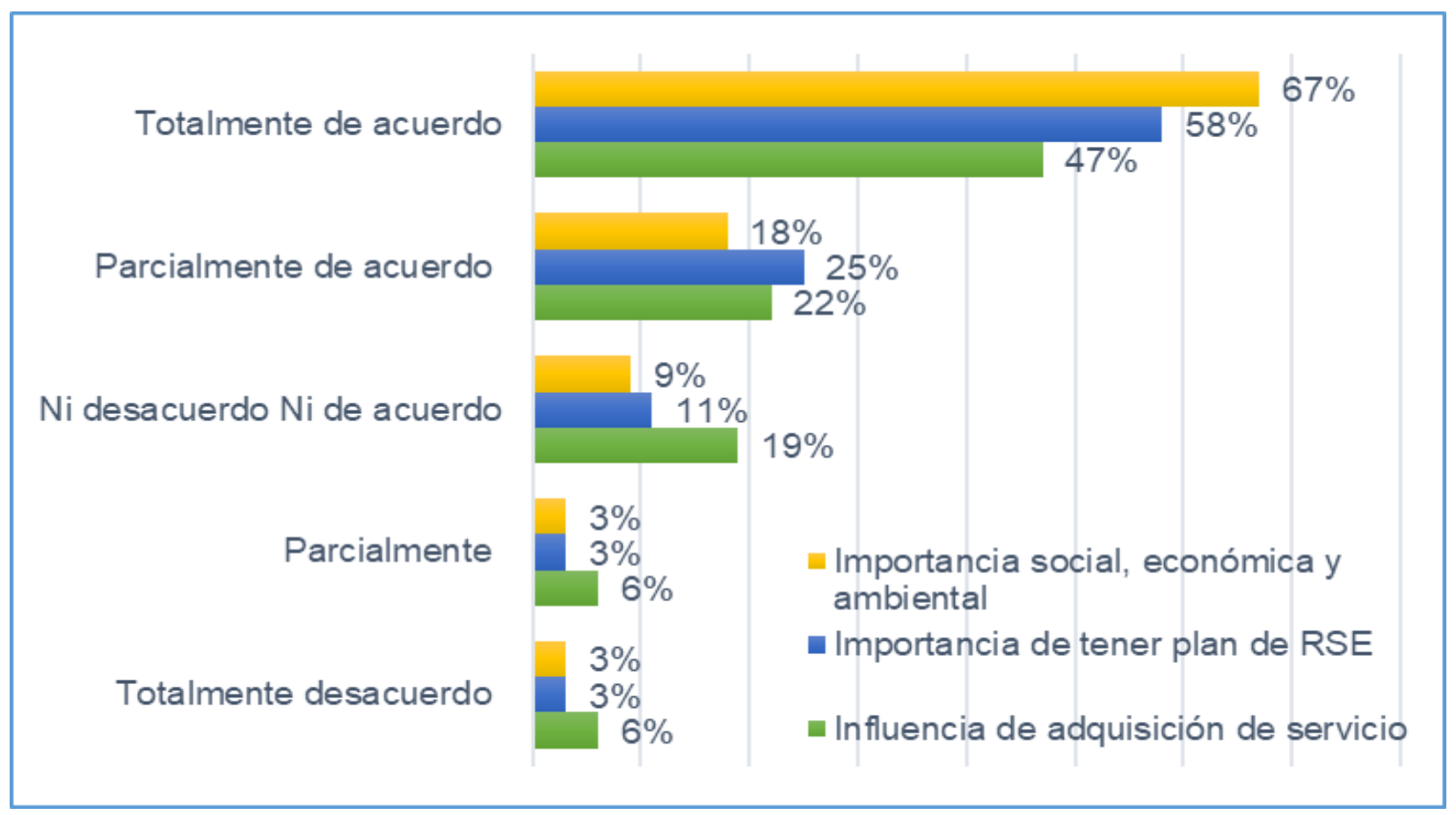

Figura 1. Importancia de la RSE en inmobiliarias. Fuente: Elaboración propia

\section{Propuesta}

Una vez realizado el análisis de la información obtenida, se crea y presenta una propuesta de plan de RSE que pueda ser introducida en empresas cuyo objetivo sea implantar esta filosofía como práctica diaria ya no como opción sino como requisito. Como punto de partida se realiza un diagnóstico para conocer la situación actual del sector en cuanto tema de RSE, luego se analiza las dimensiones que componen la responsabilidad social y para finalmente obtener el resultado último que es la sostenibilidad como alternativa para una dirección empresarial. 


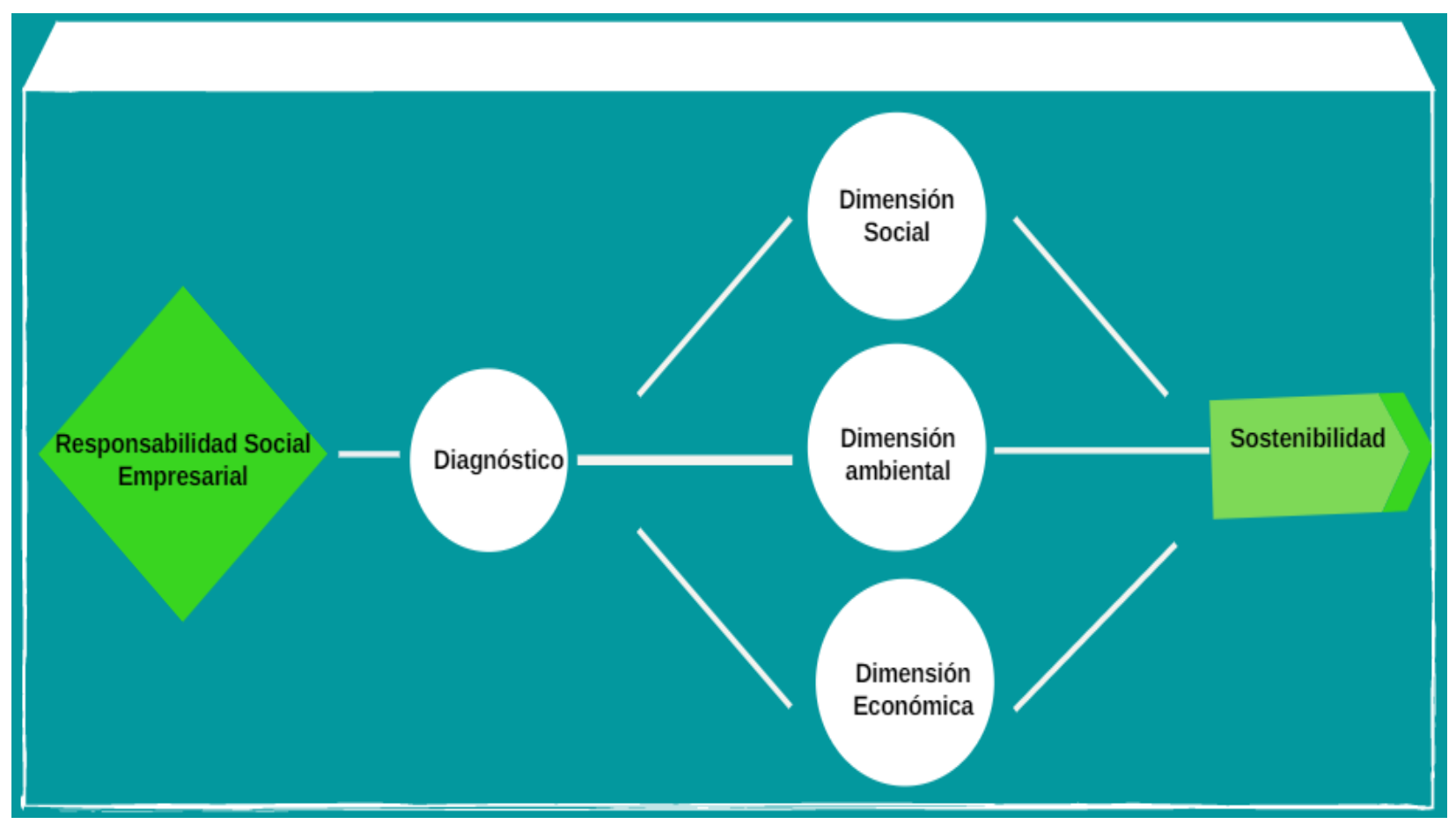

Figura 2. Propuesta de RSE. Fuente: Elaboración propia

\section{Diagnóstico}

La situación actual de la RSE en la sociedad esta priorizada debido a la importancia que esta ha tomado en la realización de las actividades de las personas. A esto se suma en que en el sector inmobiliario de la ciudad de Cuenca ninguna empresa tiene un plan de responsabilidad social que este expuesto al público, solamente se enfocan en misiones, visiones y valores generales que es la característica de la mayoría de empresas en el mercado. A continuación, esto se presenta el análisis FODA para tener el panorama situacional. 


\section{Fortalezas}

- Sector inmobiliario muy desarrollado en Cuenca

- Experiencia de la actividad en el mercado

- Conocimiento de RSE en las personas

-Aprecio por la RSE por parte de las personas

\section{Amenazas \\ - Inestabilidad política \\ - Crisis económica \\ -Emergencias sanitarias \\ -Desempleo}

\section{Debilidades}

- Falta de planes de RSE

- No existe sección encargada de RSE

-Poca difusión de servicios de inmobiliarias

- Desconfianza usuarios servicios

\section{Oportunidades}

-Amplio mercado en la ciudad de Cuenca

- Ciudad considerada una de las mejores para vivir

-Beneficios

gubernamentales para obtener inmuebles

-Ampliación urbanística de la ciudad

Figura 3. Análisis FODA. Fuente: Elaboración propia

\section{Dimensión social}

Esta se enfoca a las personas que están en el rango de acción de la empresa, se considera los factores más representativos para la imagen de las empresas o las que mayor repercusión tienen en la comunidad. Lo ideal es institucionalizar los procedimientos y debe quedar por sentado en papeles para que sean socializados y a su vez consultados cuando esto sea requerido. 


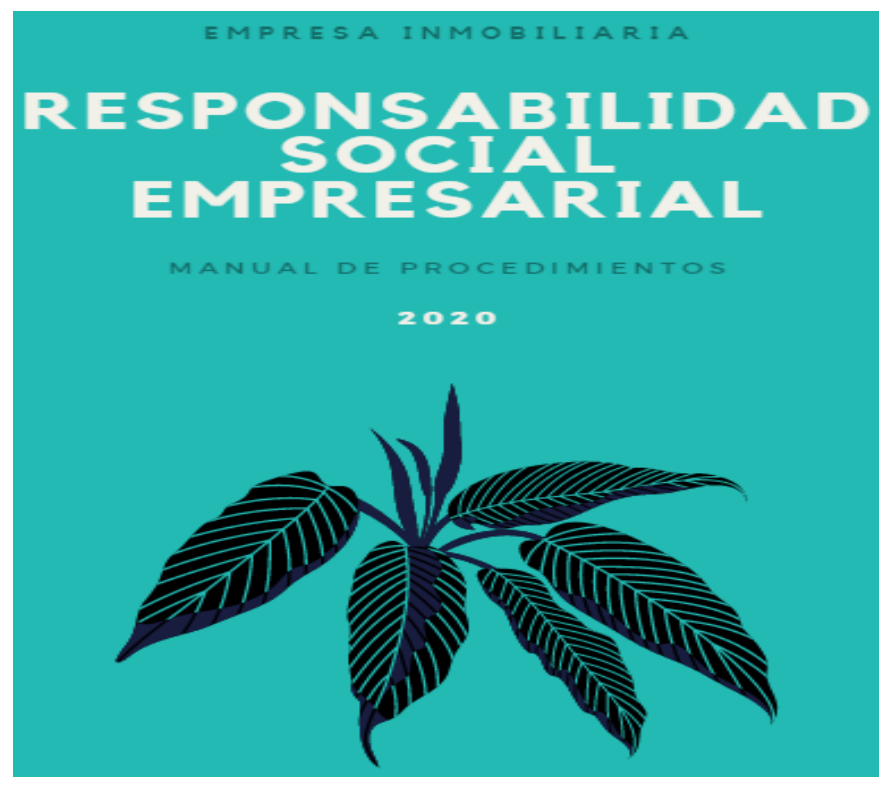

Figura 4. Manual de procedimiento de RSE. Elaboración propia

Existen elementos generales para todas las empresas y se tiene que particularizar al momento de ejecutar en las empresas inmobiliarias, con el fin desglosar o unir elementos para una mayor efectividad. En la siguiente tabla se muestra los elementos considerados.

\section{Tabla 3}

Elementos de la dimensión social

\section{Procesos}

Satisfacción en clientes

Código de ética

Proyectos sociales

\section{Subprocesos}

- Sección de quejas

- Sugerencias

- Servicio post venta

- Calidad de servicio

- Cumplimiento de acuerdos

- Publicidad verídica

- Participación de programas de interés social

- Apoyo a empleados

- Convenios gubernamentales para beneficio social

Fuente: Elaboración propia 
Dependiendo de las actividades de la empresa y los servicios que ofrezca se adecua los diferentes elementos expuestos anteriormente a la aplicación de la RSE; a continuación, se presenta una propuesta con un componente de los elementos de la dimensión social:

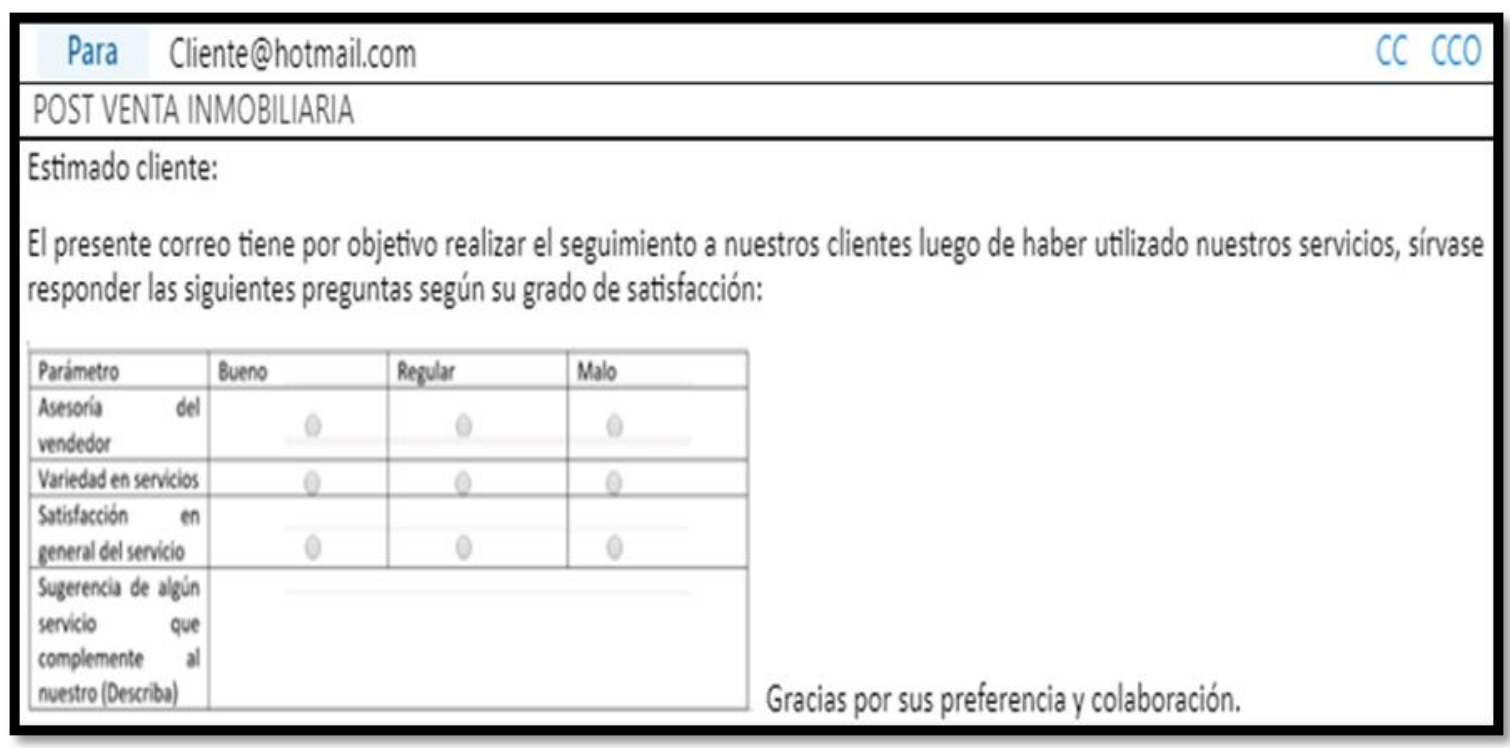

Figura 5. Satisfacción del cliente y post venta por correo electrónico.

Fuente: Elaboración propia

\section{Dimensión ambiental}

Las personas al igual que las empresas deben cuidar la naturaleza, retribuyendo lo que se utiliza para disminuir el impacto que inevitablemente causa las actividades de estas. El lugar natural de desarrollo de actividades merece especial interés, para tener proyectos a largo plazo, pues en el presente no parece ser muy importante pero el tiempo lo colocara en el sitio que verdaderamente merece. En la siguiente tabla podemos apreciar los siguientes elementos de esta dimensión: 


\section{Tabla 4}

Elementos de la dimensión ambiental

\section{Elementos}

Políticas ambientales

Indicadores de consumo

Actividades de reciclaje

Cumplimiento legal

\section{Indicadores}

- Acciones entorno a la naturaleza

- Socialización con colaboradores, clientes y sociedad

- Alianzas para alcanzar objetivos ambientales

- Reducción de consumo de agua, energía, combustibles

- Acciones para optimizar recursos

- Manejo adecuado de residuos

- Reutilización de recursos

- Programas de reciclaje

- Estudios de impactos ambientales

- Respeto por la naturaleza

- Permisos ambientales

Fuente: Elaboración propia

Estos elementos pueden ser aplicados dentro de la estructura organizacional y también con sus relacionados externos, debiendo empezar la socialización interna y de ahí será más fácil complementar las acciones con actores más allá de la propia empresa.

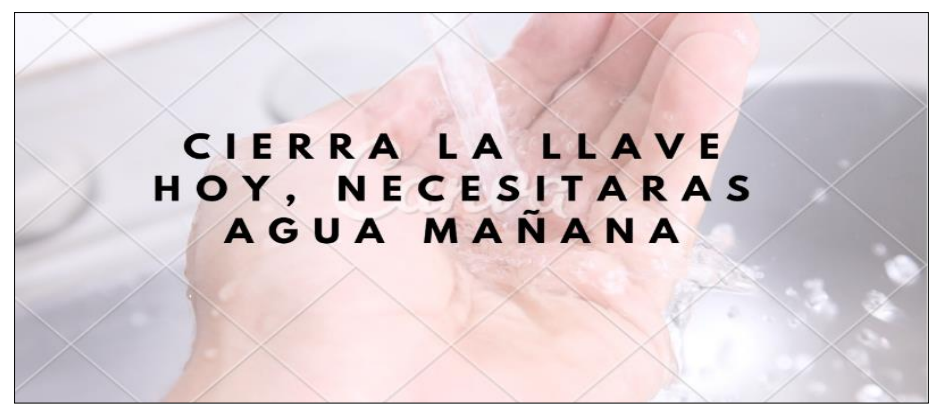

Figura 6. Anuncio para optimización del agua. Fuente: Elaboración propia 


\section{Dimensión económica}

La economía es el fin de las empresas, y en este sector no es la excepción, sin embargo, ahora se enfoca este aspecto con la cautela de cumplir todos los requisitos que siempre ha habido pero que hasta ahora son exigidos por la sociedad. Los elementos del ámbito económico se presentan en la siguiente tabla.

\section{Tabla 5}

Elementos de la dimensión económica

Elementos

Obligaciones legales

Condiciones de pagos acordados

Control en operaciones financieras

\section{Indicadores}

- Empleados

- Gobierno

- Usuarios

- Gobierno

- Proveedores

- Colaboradores

- Políticas sociales

- Transparencia en la información

- Auditorías internas y externas

Fuente: Elaboración propia

\section{Sostenibilidad}

La combinación de las dimensiones propende una sostenibilidad a largo plazo de cualquier empresa; las inmobiliarias como empresas que satisfacen necesidades básicas están destinadas a perdurar en el tiempo, pero no con los mismos actores. La actividad en el mercado está asegurada, pero depende de las políticas de RSE de las empresas ser una de las que desarrollará sus actividades a lo largo del tiempo. A continuación, se presenta la figura que genera dicha sostenibilidad. 


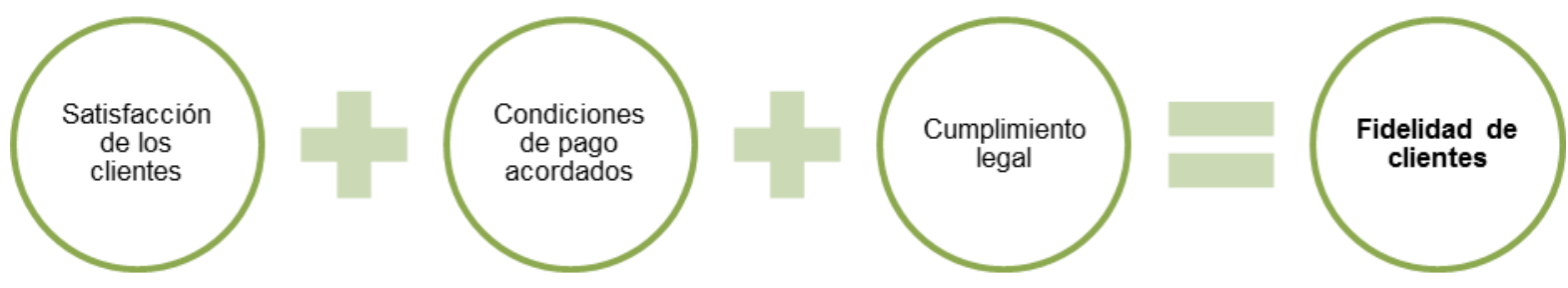

Figura 7. Relación de elementos de las dimensiones de RSE.

Fuente: Elaboración propia

Existe factores de cada dimensión que se pueden integrar entre si y formar elementos de mayor peso al momento de clasificar que empresa perdura y cual se extingue, presentamos otra figura como resultado de otra integración

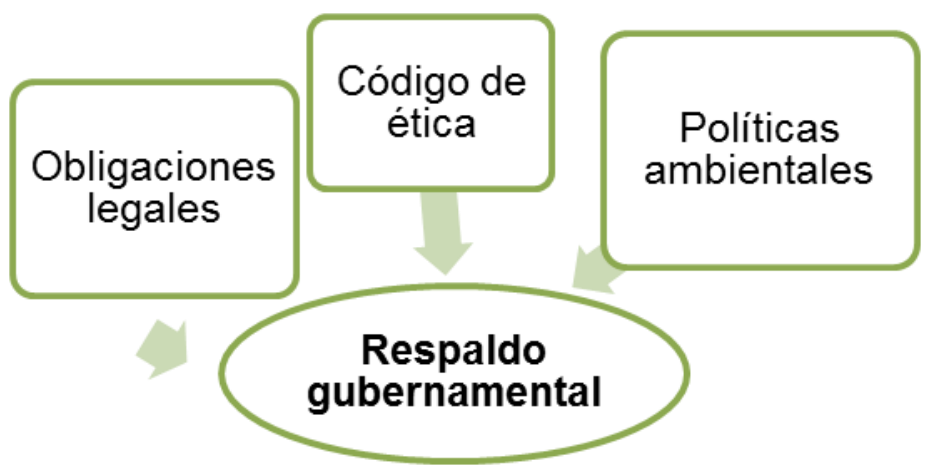

Figura 8. Relación de elementos de las dimensiones de RSE

En una figura 9, se puede destacar el beneficio en el entorno social de la empresa, que a pesar de tener interés común también demuestra una transparencia financiera. Además, añadida la preocupación ambiental con una actividad clásica se establece un soporte para el bienestar de todos en general.

Proyectos sociales

Control de operaciones financieras

Actividades de reciclaje Soctenimiento comunitario

Figura 9. Relación de elementos de las dimensiones de RSE 
La sostenibilidad de una economía, teniendo como actor principal a la empresa, es el núcleo para que todas las demás partes se acumulen y realicen esfuerzos para mantener el ciclo económico normal, entonces aquí el papel de la RSE se vuelve esencial por tener las matrices para que este acuerdo tenga resultados óptimos. La siguiente figura representa lo anteriormente descrito.

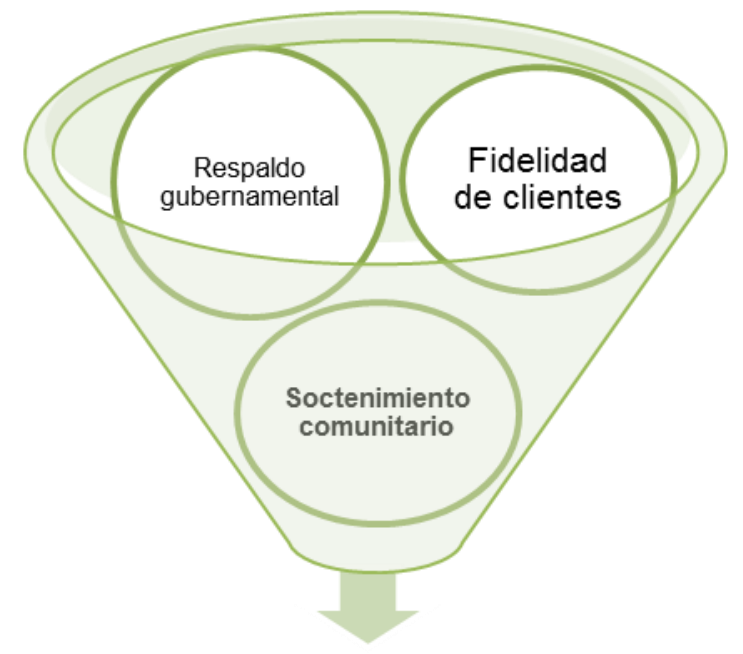

Sostenibilidad

Figura 10. Sostenibilidad

Finalmente, cabe mencionar, que las distintas combinaciones de elementos de los ámbitos sociales, económicos y ambientales dan diversos resultados, por lo que se debe obtener el mayor número de estos resultados para asegurar una RSE más confiable.

\section{CONCLUSIONES}

El sector inmobiliario en la ciudad de Cuenca tiene un amplio espacio en el mercado, por varios factores, en los que resalta tener una actividad de necesidad primaria, una población de clientes potenciales, las condiciones favorables para el desarrollo urbanístico, etc. Sin embargo, existe la necesidad imperiosa de añadir responsabilidad social empresarial de manera formal en las empresas para sacar todo el provecho que conlleva la RSE en una organización. La RSE espontanea acompañada de un respaldo legal interno y externo posibilita a las inmobiliarias a tener ventajas como la fidelización 
de clientes, elevar su imagen corporativa, mantenerse en el mercado al largo de tiempo, optimización de recursos entre otros. Un plan de RSE asertivo con las empresas fomenta la comunicación de la organización con el entorno generando nuevos beneficios a través de una socialización que explique los intereses de la empresa y la comunidad, para poder detectar el punto en donde estos intereses se unen y formen parte de un objetivo conjunto.

Finalmente al ser los servicios inmobiliarios medios para realizar fuertes inversiones, estos deben ser prestados con la mayor transparencia posible para que la desconfianza no entorpezca las transacciones comerciales; entonces que mejor que desarrollar un plan de RSE en donde todos estos aspectos y demás elementos ya tengan respuestas y lineamientos a seguir, tanto para convenios sociales, económicos y ambientales, que son el fin mismo de la RSE; de esta manera se tendrá mejores condiciones de vida empresariales e individuales, utilizando los recursos responsablemente y propendiendo un futuro sostenible para las generaciones venideras.

\section{FINANCIAMIENTO}

No monetario.

\section{AGRADECIMIENTO}

Al personal del Gobierno Autónomo Descentralizado Municipal del Cantón Cuenca Universidad Católica de Cuencas por apoyar el desarrollo de la investigación.

\section{REFERENCIAS CONSULTADAS}

Aguilera, A., \& Puerto, D. (2012). Crecimiento empresarial basado en la Responsabilidad Social. [Business growth based on Social Responsibility]. Pensamiento y Gestión, 32; 1-26. Recuperado de: https://n9.cl/9otv

Alvarado, A., \& Schlesinger, M. (2008). Dimensionalidad de la responsabilidad social empresarial percibida y sus efectos sobre la imagen y la reputación: Una aproximación desde el modelo de Carroll. [Dimensionality of perceived corporate social responsibility and its effects on image and reputation: An approach from the Carroll model]. Estudios Gerenciales, 24(108); 37-59. Recuperado de https://n9.cl/gyea9 
Andía-Valencia, W. (2015). La responsabilidad social: análisis del enfoque de ISO 26000. [Social responsibility: analysis of the ISO 26000 approach]. Industrial Data, 18(2), 55-60. https://doi.org/10.15381/idata.v18i2.12097

Avendaño, W. (2013). Responsabilidad social (RS) y responsabilidad social corporativa (RSC): una nueva perspectiva para las empresas. [Social Responsibility (SR) and Corporate Social Responsibility (CSR): a new perspective for companies]. Reviste Lasallista de investigacion, 10(1); 152-163. Recuperado de https://n9.cl/hoy4

Barbachan, M. (2017). La Responsabilidad Social Empresarial en el Perú: Desafíos y Oportunidades. [Corporate Social Responsibility in Peru: Challenges and Opportunities]. InnovaG, (2), 56-62. Recuperado de: https://n9.cl/8n5h6

Caridad, M., Hernández, P., \& De-Pelekais, C. (2014). Responsabilidad gerencial: elemento integrador de la sustentabilidad en la responsabilidad social empresarial. [Management responsibility: integrating sustainability element into corporate social responsibility]. Opción, 30(75); 35-54. Recuperado de https://n9.cl/247h5

Cestagalli, L., Téllez, H., \& Cuevas, S. (2016). La responsabilidad social empresarial: una ventaja competitiva en empresas de cobranza y call center. [Corporate social responsibility: a competitive advantage in collection and call center companies]. CRITERIOS, 9(2); 107-127. Recuperado de https://n9.cl/78qpn

Chaves-Ávila, R., \& Monzón-Campos, J. (2018). La economía social ante los paradigmas económicos emergentes: innovación social, economía colaborativa, economía circular, responsabilidad social empresarial, economía del bien común, empresa social y economía solidaria. [The social economy in the face of emerging economic paradigms: social innovation, collaborative economy, circular economy, corporate social responsibility, economy of the common welfare, social enterprise and solidarity economy]. CIRIEC-España, Revista De Economía Pública, Social y Cooperativa, 93, 5-50. https://doi.org/10.7203/CIRIECE.93.12901

Comisión de la Comunidades Europeas. (2001). Fomentar un marco europeo para la responsabilidad social de las empresas. [Promoting a European framework for corporate social responsibility]. Recuperado de https://n9.cl/j76u

Correa, J. (2007). Evolución Histórica de los Conceptos de Responsabilidad Social Empresarial y Balance Social. [Historical Evolution of the Concepts of Corporate Social Responsibility and Social Balance]. Semestre Económico, 10(20); 87-102; Recuperado de https://n9.cl/3sjm 
Cortés-Mura, H., \& Peña-Reyes, J. (2015). De la sostenibilidad a la sustentabilidad. Modelo de desarrollo sustentable para su implementación en políticas y proyectos. [From sustainability to maintainability. Sustainable development model for its implementation in policies and projects]. Revista Escuela De Administración De Negocios, (78), 40-54. https://doi.org/10.21158/01208160.n78.2015.1189

Del-Cid, A., Méndez, R., \& Sandoval, F. (2011). Investigación. Fundamentos y Metodología. [Research. Fundamentals and Methodology]. México: Pearson Eucación. Recuperado de: https://n9.cl/xspa

Duarte, F. (2015). Responsabilidad social empresarial. [Corporate Social Responsibility]. Recuperado de https://n9.cl/5ozl7

Duque, Y., Cardona, M., \& Rendón, J. (2013). Responsabilidad Social Empresarial: Teorías, índices, estándares y certificaciones. [Corporate Social Responsibility: Theories, Indexes, Standards and Certifications]. Cuadernos de Administración, 20(50); 196-206. https://doi.org/10.25100/cdea.v29i50.55

Gallardo-Vázquez, D., \& Sánchez-Hernández, M. I. (2013). Análisis de la incidencia de la responsabilidad social empresarial en el éxito competitivo de las microempresas y el papel de la innovación. [Analysis of the corporate social responsibility's impact on the competitive success of micro-enterprises and the innovation role]. UCJC Business and Society Review, 14-31.Recuperado de https://n9.cl/7cag2

García-Marzá, D. (2017). De los códigos a las auditorías éticas: una infraestructura ética para la comunicación de la responsabilidad social. [From codes to ethical audits: an ethical infrastructure for communicating social responsibility]. El profesional de la información, 26(2); 268-276. Recuperado de https://n9.cl/1k1p

Gobierno Autónomo Descentralizado Municipal del Cantón Cuenca. (2015). Plan de Desarrollo y Ordenamiento Territorial del Cantón Cuenca. [Development and land management plan of Cuenca Canton]. Recuperado de https://n9.cl/8vaw

González-Bustos, J. P., Narváez-Zurita, C. I., \& Erazo-Álvarez, J. C. (2020). La gestión de responsabilidad social de las cooperativas de ahorro y crédito de la provincia del Azuay y su retorno financiero [Social responsibility management of the savings and credit cooperatives and its financial return in Azuay province]. Dominio de las Ciencias, 04-33

Henríquez, R., \& Oreste, R. (2015). Implicancias de una Responsabilidad Social Empresarial Sustentable. [Interventions of Sustainable Corporate Social Responsibility]. Revista Electrónica Gestión de las Personas y Tecnología, 8(23); 16-27. Recuperado de https://n9.cl/oa45 
Hernández, R., Fernández, C., \& Baptista, M. (2014). Metodología de la Investigación. [Research Methodology]. Mexico DF: McGraw-Hill / Interamericana Editores, S.A. DE C.V

Koehn, P., \& Fierro-Ulloa, I. (2015). El modelo de responsabilidad social empresarial como estrategia en el sector inmobiliario en el Ecuador. [The model of corporate social responsibility as a strategy in the real estate sector in Ecuador]. Saber, Ciencia Y Libertad, 10(1), 101-114. https://doi.org/10.18041/23823240/saber.2015v10n1.875

Maldonado-Calero, J., \& Viteri-Martínez, M. (2017). Contabilidad, responsabilidad social corporativa y sostenibilidad. [Accounting, corporate social responsibility and sustainability]. Revista Publicando, 3(9), 579-588. Recuperado de https://n9.cl/dw15

Medina-Giacomozzi, A., \& Severino-González, P. (2014). Responsabilidad empresarial: generación de capital social de las empresas. [Corporate responsibility: generating corporate social capital]. Contabilidad Y Negocios, 9(17), 63-72. Recuperado de https://n9.cl/njw3

Mendoza, M., Hernández, A., \& García, M. (2013). Responsabilidad Social. [Social Responsibility]. Contribuciones a la Economía, 1-19. Recuperado de https://n9.cl/wre4

Molero-García, G. (2016). La responsabilidad social empresarial en el contexto del capital social. [Corporate social responsibility in the context of social capital]. Omnia, 22(3); 46-59. Recuperado de https://n9.cl/1c1h6

Mora-Mayoral, M. J., \& Martínez-Martínez, F.R. (2018). Desarrollo local sostenible, responsabilidad social corporativa y emprendimiento social. [Sustainable local development, corporate social responsibility and social entrepreneurship]. Equidad y Desarrollo, (31), 27-46. https://doi.org/10.19052/ed.4375

Reyno-Momberg, M. (2006). Responsabilidad Social Empresarial (RSE) Como Ventaja Competitiva. [Corporate Social Responsibility (CSR) as a Competitive Advantage]. Recuperado de https://n9.cl/q272

Rodríguez, D., Erazo, J., \& Narváez, C. (2019). Técnicas cuantitativas de investigación de mercados aplicadas al consumo de carne en la generación millennial de la ciudad de Cuenca (Ecuador) [Quantitative market research techniques applied to meat consumption in the millennial generation of Cuenca city (Ecuador)]. Revista Espacios, 40(32); 20. Recuperado de: https://url2.cl/ibtfw

Valarezo-González, K, \& Túñez-López, J. (2014). Responsabilidad Social Universitaria. Apuntes para un modelo de RSU. [University Social Responsibility. Notes for an MSW model]. Recuperado de: https://n9.cl/5i07 
Vélez, X., \& Cano, E. (2016). Los diferentes tipos de responsabilidad social y sus implicaciones éticas. [The different types of social responsibility and their ethical implications]. Dominios de las Ciencias, 2(especial); 117-126. Recuperado de: https://n9.cl/8hnw

Waddock, S. (2004). Parallel universes: Companies, academics, and the progress of corporate citizenship. [Universos paralelos: empresas, académicos y el progreso de la ciudadanía corporativa]. Business and Society Review, 109(1); 5-42. Recuperado de https://n9.cl/ov787

(C2020 por los autores. Este artículo es de acceso abierto y distribuido según los términos y condiciones de la licencia Creative Commons Atribución-NoComercial-Compartirlgual 4.0 Internacional (CC BY-NC-SA 4.0) (https://creativecommons.org/licenses/by-nc-sa/4.0/). 